\title{
It Is Tough to Make Predictions, Especially about the Future ${ }^{1}$
}

\author{
M. Bezzi ${ }^{a} \quad$ M. Noppen ${ }^{b}$ \\ aSpedali Civili di Brescia, Brescia, Italy; ${ }^{b}$ University Hospital UZ Brussel, Brussels, Belgium
}

End-stage emphysema is a horrible because incurable and invalidating disease. Prior to the advent of lung transplantation - which is in fact the only definitive but obviously very limited therapy - surgeons have tried to improve pulmonary function by resecting (the most diseased) portions of the lung. Essentially, this lung volume reduction surgery (LVRS) is thought to improve matching of the size of the lung to the size of the hemithorax containing it, and to allow the space previously occupied by the sickest lung to be reclaimed by more normal lung [1]. Although all of this might be true, the bottom line question is: did it make patients better? Because of the amazing power of expectations of patients as well as physicians in new treatments and techniques, controlled trials (preferably with sham procedures in the case of device trials) are mandatory [2]. After a few decades of 'wild' LVRS, a properly controlled (albeit not sham-controlled) prospective randomized trial [3] showed that there was indeed some true clinical benefit to be expected from LVRS, but only in a (post-hoc) well-defined subgroup of patients, with hyperinflation, severe but not-too-severe diffusion capacity decrease, heterogeneously upper-lobe distributed emphysema and a decreased exercise capacity; in all other patients, LVRS was not only useless, but even harmful, taking into account its huge morbidity, cost and even mortality. LVRS has since become an exceptional treatment modality [4].

\section{KARGER}

Fax +41613061234 E-Mail karger@karger.ch www.karger.com
(C) 2010 S. Karger AG, Basel

0025-7931/10/0805-0369\$26.00/0

Accessible online at:

www.karger.com/res
Three lessons can be learned from this: first, there is a need for a less invasive and preferably reversible technique for lung volume reduction. Secondly, there is a need for prospective, randomized and (preferably sham) control of these techniques, and thirdly, there is a need for an a priori selection of patients, to predict the future.

So, are there less invasive techniques out there?

Interventional pulmonologists, surgeons, investigators, small venture capital firms as well as big device and instrument companies have come up with a variety of minimally invasive techniques and devices. These can be roughly categorized $[5,6]$ as reversible blocking devices, including endobronchial blockers or plugs such as the silicone plug described by Watanabe et al. [7] and endobronchial valves such as the Zephyr ${ }^{\circledR}$ valve (Pulmonx Inc., Palo Alto, Calif., USA) [8], the Miyazawa silicone valve from Novatech, La Ciotat, France [9], or the valve from Spiration, Redmond, Wash., USA [10, 11], reversible non-blocking devices (lung volume reduction coils of PneumRx, Mountain View, Calif., USA) [12], and irreversible non-blocking techniques (bronchoscopic thermal vapor ablation using the apparatus from Uptake Medical, Seattle, Wash., USA [13], and the polymeric lung

\footnotetext{
1 Quote by Lawrence Peter 'Yogi' Berra, American baseball legend and famous for his witty quotes and comments.
}

Marc Noppen, MD, PhD, CEO

University Hospital UZ Brussel

101 Laarbeeklaan

BE-1090 Brussels (Belgium)

Tel. +32 2477 5501, Fax +32 2477 5515, E-Mail marc.noppen@uzbrussel.be 
volume reduction system of Aeris Therapeutics, Woburn, Ma., USA [14]) for heterogeneous disease, as well as the endobronchial airway bypass system of Bronchus, Mountain View, Calif., USA [15], or direct transthoracic pulmonary drainage [16] for homogeneous disease.

Secondly, do patients benefit from these techniques and devices? In other words, do we have controlled data? Very little, unfortunately. Although numerous singlecenter feasibility, safety and preliminary outcome data are available, only one large, prospective, randomized, best care controlled trial (VENT trial) has been finished, but has not yet been published as a complete, full paper. However, the preliminary release of data shows a small overall 'mean' response for the patient group as a whole, but quite important and clinically relevant improvements in a subgroup of patients (highly heterogeneous emphysema, complete lobar fissures and technically and anatomically complete airway occlusion, and hence, lobar exclusion with atelectasis). Such 'categorical' results showing clinical benefit only to a restricted subgroup of patients seem to occur in all other volume reduction techniques and devices used in heterogeneous disease. Why is this? Probably the major reason is that 'lung volume reduction by means of lobar atelectasis' is counteracted by the variable presence of interlobar collateral ventilation [6]. (However, to make meaningful conclusions, we still have to wait for the full results of current large-scale studies.)

Thirdly, how can we - with our current knowledge predict the future, i.e. predict which technique to use in which patient, and with what purpose? We think it is fair to say that for homogeneous disease, we currently do not know and have to wait for the results of the EASE trial, a large, multicenter, randomized, sham-controlled trial, the publication of which is expected in the course of 2010. The Brazilian experiment [16] is interesting but very unlikely to be applicable at a large scale. In heterogeneous disease, we still do not know. With the pending publication of the 2 largest trials (VENT trial, to be fully published; Spiration trial, to be fully published), it will be necessary to provide a good a priori probability of success, e.g., to obtain reimbursement. In Belgium, for instance, reimbursement has been put on hold pending such reliable 'a priori patient selection' methods [17]. Predicting the probability of post-valve placement atelectasis - if this would be the goal of treatment - seems to become inevitable, and for this, we need 'a simple and accurate pretreatment selection procedure', as was predicted already 3 years ago [6]. In this issue of Respiration, Gompelmann et al. [18] from the extremely productive
Heidelberg group describe their experience with the Chartis ${ }^{\circledR}$ system (Pulmonx), a minimally invasive flexible bronchoscopic catheter technique in spontaneously breathing patients, allowing continuous measurements of air pressures and flows distal from lobar airway balloon occlusion, hence allowing the measurement of collateral ventilation in the occluded lobe. This is an improvement in comparison with previously available more cumbersome or less physiological and potentially more harmful (because of the use of positive pressures) in vivo techniques to measure collateral ventilation [6]; the principle of this method has been validated by Aljuri and Freitag [19].

In this study, Gompelmann et al. [18] show that the pretreatment use of this system was feasible in 21 out of 25 patients (84\%), and that in the 20 'eligible' patients, the system accurately predicted treatment success or not (i.e. it predicted the best known proxy of success, lobar atelectasis) in 18 patients (90\%). Of course, these data are to be handled with care. First, and unfortunately, it is a bit of a messy paper. The authors define 2 study endpoints: (1) safety and (2) feasibility of obtaining measurements (which, thirdly, would be predictive for atelectasis), so there are actually 3 endpoints. The first endpoint (safety) actually described the safety of the subsequent volume reduction procedure. In fact, we learned nothing about safety of the Chartis procedure, nor about technical data (how long did the measurements take? Were there differences between upper and lower lobe measurements? What did the patients experience?). The second endpoint was feasibility to obtain adequate measurements. This was correctly given as $84 \%$. The authors might have elaborated more on the reasons why in 1 patient out of 7, measurement was impossible. For instance, all measurement failures occurred in the first half of the patients studied (learning curve effect?). Also, there are no data on patient selection (were these consecutive patients? Was there a group of patients in which the authors first 'tried out' the device? Did all investigators experience similar results?). Finally, the vast content of the paper discusses the actual accuracy of the system in predicting atelectasis or not ('match or mismatch'). In this setting, it would have been preferable to apply an intention-to-treat analysis instead of a post-hoc elimination of otherwise uneligible patients. In that case, the system would have enabled a correct prediction in $18 / 25$ cases, that is $74 \%$ ( 3 out of 4 ), which is not bad, but I think not good enough for the payers [17]. Furthermore, a significant number of patients respond to endobronchial valve placement symptomatically and objectively even without atelectasis, i.e. mainly by reduction in 
static and/or dynamic hyperinflation [20] and interlobar shift of ventilation [21]; therefore, relying on this technique would exclude a number of patients potentially benefiting from endobronchial valve treatment.

Nevertheless, it is a good start. This experience needs to be repeated in a larger and multicentric setting, using an intention-to-treat analysis design. Only then might it be possible to oppose Yogi Berra in actually predicting the future by selecting a priori which patients would benefit beyond reasonable doubt of endobronchial valve placement.

\section{References}

1 Fessler HE, Scharf SM, Ingenito EP, McKenna RJ, Sharafkhaneh A: Physiologic basis for improved pulmonary function after lung volume reduction. Proc Am Thorac Soc 2008;5:416-420.

-2 Sutherland ER: Sham procedure versus usual care as the control in clinical trials of devices. Which is better? Proc Am Thorac Soc 2007;4:574-576.

$\checkmark 3$ NETT Research Group: A randomized trial comparing lung-volume reduction surgery with medical therapy for severe emphysema. N Engl J Med 2003;348:2059-2073.

4 Chang AC, Chan KM, Martinez FJ: Lessons from the NETT trial. Semin Thorac Cardiovasc Surg 2007;19:172-180.

5 Herth FJF, Gompelmann D, Ernst A, Eberhardt R: Endoscopic lung volume reduction. Respiration 2010;79:5-13.

6 Noppen M: Collateral ventilation in endstage emphysema. A blessing or a curse for new bronchoscopic treatment approaches (or both)? Respiration 2007;74:493-495.

7 Watanabe Y, Matsuo K, Tamaoki A, Komoto R, Hiraki S: Bronchial occlusion with endobronchial Watanabe spigot. J Bronchol 2003; 10:264-267.

$>_{8}$ Strange C, Herth FJF, Kovitz KL, McLennan G, Goldin J, Noppen M, Criner GJ, Sciurba FC: Design of the Endobronchial Valve for Emphysema Palliation Trial (VENT): a nonsurgical method of lung volume reduction. BMC Pulm Med 2007;7:10.
9 Galluccio G, Lucantoni G: Bronchoscopic lung volume reduction for pulmonary emphysema: preliminary experience with a new Novatech $^{\circledR}$ endobronchial silicone one-way valve. Interact Cardiovasc Thorac Surg, Epub ahead of print.

10 Springmeyer SC, Bolliger CT, Waddell TK, Gonzalez X, Wood DE: IBV valve trial research teams: treatment of heterogeneous emphysema using the spiration IBV valves. Thorac Surg Clin 2009; 19:247-253.

11 Sterman DH, Mehta AC, Wood DE, Mathur PN, McKenna RJ Jr, Ost DE, Truwit JD, Diaz P, Wahidi MM, Cerfolio R, Maxfield R, Musani AI, Gildea T, Sheski F, Machuzak M, Haas AR, Gonzalez HX, Springmeyer SC, IBV Valve US Pilot Trial Research Team: A multicenter pilot study of a bronchial valve for the treatment of severe emphysema. Respiration 2010;79:222-233.

12 Herth FJF, Eberhardt R, Ernst A: Pilot study of an improved lung volume reduction coil for the treatment of emphysema. Am J Respir Crit Care Med 2009;179:A6160.

13 Snell GI, Hopkins P, Westall G, Holsworth L, Carle A, Williams TE: A feasibility and safety study of bronchial thermal vapour ablation: a novel emphysema therapy. Ann Thorac Surg 2009;88:1993-1998.

14 Herth FJF, Stanzel F, Magnussen H, Ernst A, Eberhardt R: A dose ranging study of the Aeris polymeric lung volume reduction system in patients with advanced upper lobe predominant emphysema. Eur Respir J 2009; 35:A1828. $\checkmark 15$ Moore AJ, Cetti E, Hai-Yahia S, Carby M, Bjorling G, Karlsson S, Shah P, Goldstraw P, Moxham J, Jordan S, Polkey MI: Unilateral extra airway bypass in advanced emphysema. Ann Thorac Surg 2010;89:899-906.

16 Saad R Jr, Dorgan Neto V, Botter M, Stirbulov R, Rivabern JH, Gonçalves R: Therapeutic application of collateral ventilation with pulmonary drainage in the treatment of diffuse emphysema: report of the first three cases. J Bras Pneumol 2009;35:14-19.

17 Anonymous: Endobronchial valves in the treatment of emphysema: a rapid Health Technology Assessment. Brussels, Federal Knowledge Centre, KCE Report 114A, 2009.

18 Gompelmann D, Eberhardt R, Michaud G, Ernst A, Herth FJF: Predicting atelectasis by assessment of collateral ventilation prior to endobronchial lung volume reduction: a feasibility study. Respiration 2010;80:419-425.

19 Aljuri N, Freitag L: Validation and pilot clinical study of a new bronchoscopic method to measure collateral ventilation before endobronchial lung volume reduction. J Appl Physiol 2009; 106:774-783.

20 Ingenito EP, Wood DE, Utz JP: Bronchoscopic lung volume reduction in severe emphysema. Proc Am Thorac Soc 2008;5:454466.

21 Coxson HO, Nasute Fauerbach PV, StornessBliss C, Muller NL, Cogswell S, Dillard DH, et al: The computed tomography assessment of lung volume changes after bronchial valve treatment. Eur Respir J 2008;32:1443-1450. 